\title{
Bloch vector, disclination and exotic quantum holonomy
}

\author{
Atushi Tanaka ${ }^{\mathrm{a}}$, Taksu Cheon ${ }^{\mathrm{b}}$ \\ ${ }^{a}$ Department of Physics, Tokyo Metropolitan University, Hachioji, Tokyo 192-0397, Japan \\ ${ }^{b}$ Laboratory of Physics, Kochi University of Technology, Tosa Yamada, Kochi 782-8502, Japan
}

\begin{abstract}
A topological formulation of the eigenspace anholonomy, where eigenspaces are interchanged by adiabatic cycles, is introduced. The anholonomy in two-level systems is identified with a disclination of the director (headless vector) of a Bloch vector, which characterizes eigenprojectors. The covering map structure behind the exotic quantum holonomy and the role of the homotopy classification of adiabatic cycles are elucidated. The extensions of this formulation to nonadiabatic cycles and $N$-level systems are outlined.
\end{abstract}

\section{Introduction}

An adiabatic cycle, where an external parameter of a sys$\sim$ tem is varied infinitely slowly along a closed path, may induce a nontrivial change, which is sometimes referred to as an anholonomy. A famous example is the geometric phase in stationary states [1]. This is also called as a phase holonomy, which is derived from the interpretation in terms of the differential geometry [2]. Besides the phase of state vector, it turned out that (quasi-)eigenenergies and eigenspaces of stationary states of a closed quantum system may exhibit anholonomies [3]. We call such a change an exotic quantum holonomy.

A more precise description of the exotic quantum holonomy is the following. Suppose that a system is initially in a stationary state, and undergo a unitary time evolution induced by an adiabatic cycle. The final state of the system is orthogonal to the initial state. In accordance to the correspondence between eigenstates and eigenenergies, the trajectory of the eigenenergy connects two different eigenenergies of the initial system. In other words, the adiabatic cycle interchanges the eigenspaces and eigenenergies [3].

Note that the exotic quantum holonomy is different from Wilczek-Zee's holonomy [4], since the former occurs even when there is no spectral degeneracy. Also note that the exotic quantum holonomy is different from the interchange, or the flip, of eigenvectors induced by a cycle around a non-Hermitian degeneracy point [5], which is known as Kato's exceptional point (EP) [6, 7], in spite of their resemblance explained below. This is because the decay due to the non-Hermiticity makes the stringent limitation to observe the flip in the adiabatic limit [5, 8]. Namely, the flip due to EPs is observable only in the nonunitary time evolution whose timescale is shorter than the relevant lifetimes in the unstable system, and, in the parametric evolution [9, 10].

URL: http://researchmap.jp/tanaka-atushi/ (Atushi Tanaka), http://researchmap.jp/T_Zen/ (Taksu Cheon)
The earliest example of the exotic quantum holonomy is the one-dimensional particle under a generalized point potential [3, 11, 12]. Since then, examples are found in quantum maps [13, 14], quantum circuits [15], and quantum graphs [16[18]. It is also shown that the Lieb-Liniger model [19, 20], which describes one-dimensional Bose systems, exhibits the exotic quantum holonomy along a cycle made of the confinement induced resonance [21, 22], whose experimental realization should be feasible within the current state of the art [23]. As an application, an acceleration of the adiabatic quantum computation was examined [24].

There are several theoretical works on the exotic quantum holonomy. Firstly, Fujikawa's gauge theoretical formulation for the phase holonomy [25, 26] is extended so as to incorporate the eigenspace anholonomy, which is understood as a holonomy of basis vectors [27, 28]. Secondly, Viennot proposed another gauge theoretical formulation based on the adiabatic Floquet theory, where the nontrivial Floquet block change is discussed in terms of gerbes [29]. Thirdly, the exotic quantum holonomy is associated to the state flip induced by EPs through the generalized Fujikawa formalism [30, 31]. Here the analytic continuation of a Hermitian (or unitary) adiabatic cycle provides non-Hermitian cycles, which relate the Riemann surface structure of eigenenergies [32] to the exotic quantum holonomy.

In spite of these efforts, there still remain puzzling points on the nature of the exotic quantum holonomy. Firstly, the eigenspace anholonomy and the phase holonomy are mixed in the generalized Fujikawa formalism, and there is only an ad hoc procedure to disentangle them [33]. Also, it is not straightforward to extract the geometrical picture from the generalized Fujikawa formalism. Although a solution to this problem is given by Simon's formulation as for the phase holonomy [2], there has been no known counterpart of the Simon's formulation for the exotic quantum holonomy. Secondly, Viennot's theory is for periodically driven systems and, is not applicable to the examples of the exotic quantum holonomy in autonomous (Hamiltonian) systems [18, 23, 34]. Thirdly, the association of the exotic quantum holonomy to the non-Hermitian degeneracy 

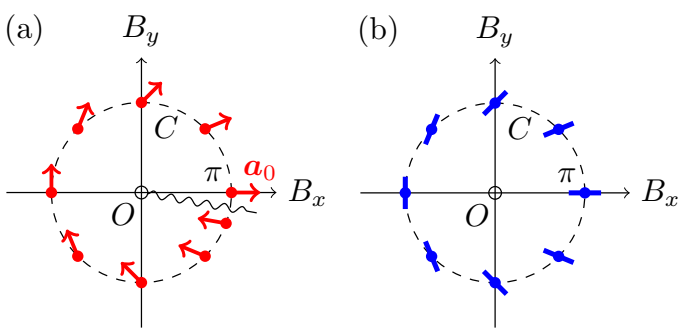

Figure 1: A disclination of eigenobjects of quantum kicked spin- $\frac{1}{2}$ (Eq. (1) in the $\left(B_{x}, B_{y}\right)$-plane. (a) The Bloch vector $\boldsymbol{a}$ (Eq. (4)) at a circle $C(|\boldsymbol{B}|=\pi)$. The Bloch vector is not well-defined at the origin, which reflects the multiplevaluedness of $\boldsymbol{a}$. A "branch cut" is depicted by a wavy line. Here $\boldsymbol{a}_{0}=\boldsymbol{e}_{x}$ is the normalized Bloch vector at the initial point $(\pi, 0)$ of $C$. The adiabatic time evolution along $C$ induces a flip of $\boldsymbol{a}$. (b) The director (headless vector) $\boldsymbol{n}$ of $\boldsymbol{a}$ at the circle $C$. Since $\boldsymbol{n}$ is single-valued in the $\left(B_{x}, B_{y}\right)$-plane, no branch cut needs to be drawn. Still, the line defect remains at the origin.

requires the analyticity of the adiabatic parameter. However, it is easy to construct the examples that lack the analyticity [18]. Hence the EP interpretation of the exotic quantum holonomy is applicable only to a limited class of examples.

In this manuscript, we introduce a topological formulation of the exotic quantum holonomy, in particular the eigenspace anholonomy, to clarify these problems. This enables us to identify the quantity that predicts whether a given cycle exhibits the eigenspace anholonomy. The first key concept in our approach is the ordered set of eigenprojectors. We regard the eigenspace anholonomy as a permutation, which is induced by an adiabatic cycle, among the elements of the ordered set of eigenprojectors. This may be considered as a counterpart to Simon's vector bundle formulation of the phase holonomy [2]. In a two-level system, we show that a permutation occurs only when the adiabatic cycle encloses a singular point odd times. The second key concept in our topological formulation is the cycles in a quantum dynamical variable that takes the place of conventional cycles in adiabatic parameters. It is shown that the topological nature of the eigenspace anholonomy has a direct link with the homotopy classification of cycles [35]. For example, the singular point mentioned above may be called a disclination, or line defect [36]. Furthermore, in common with Aharonov-Anandan formulation [37], the new definition of the cycles allows us to extend the eigenspace anholonomy to nonadiabatic cycles.

\section{Quantum kicked spin- $\frac{1}{2}$ with two adiabatic parameters}

Throughout the presentation of our formulation, we assume that the systems are described either by Hermitian Hamiltonians or by unitary Floquet operators. We also assume that there is no spectral degeneracy in the adiabatic cycles. For concreteness, we employ a periodically driven spin- $\frac{1}{2}$ to illustrate our formulation, which is immediately applicable to an arbitrary two-level system. An extension to an arbitrary $N$-level system is also to be shown.

Let us suppose that the system is described by a time-periodic Hamiltonian $\hat{H}(t) \equiv \frac{1}{2} \boldsymbol{B} \cdot \hat{\boldsymbol{\sigma}}+\frac{1}{2} \lambda\left(1-\hat{\sigma}_{z}\right) \sum_{m=-\infty}^{\infty} \delta(t-m)$,

${ }^{1}$ The exotic quantum holonomy in an autonomous Hamiltonian system re- where $\boldsymbol{B}$ is a static magnetic field and $\hat{\boldsymbol{\sigma}} \equiv \boldsymbol{e}_{x} \hat{\sigma}_{x}+\boldsymbol{e}_{y} \hat{\sigma}_{y}+\boldsymbol{e}_{z} \hat{\sigma}_{z}$ is a unimodular linear combination of Pauli matrices $\hat{\sigma}_{j}(j=$ $x, y, z)$. The Hamiltonian $\hat{H}(t)$ contains a periodically pulsed rank-1 perturbation [38] with strength $\lambda$.

In the following, $\boldsymbol{B}$ is assumed to be restricted within $x y$ plane, i.e., $\boldsymbol{B}=B_{x} \boldsymbol{e}_{x}+B_{y} \boldsymbol{e}_{y}$. To parameterize $\boldsymbol{B}$ with the cylindrical coordinates $B$ and $\phi$, we introduce $\boldsymbol{e}_{\rho} \equiv \boldsymbol{e}_{x} \cos \phi+\boldsymbol{e}_{y} \sin \phi$ and $\boldsymbol{e}_{\phi} \equiv \boldsymbol{e}_{y} \cos \phi-\boldsymbol{e}_{x} \sin \phi$. Hence we have $\boldsymbol{B}=B \boldsymbol{e}_{\rho}$. We also impose $\lambda=\phi$ in the following. We introduce a Floquet operator, which describes a unit time evolution generated by $\hat{H}(t)$ :

$$
\hat{U} \equiv \exp \left(-i \phi \frac{1-\hat{\sigma}_{z}}{2}\right) \exp \left(-\frac{i}{2} B \boldsymbol{e}_{\rho} \cdot \hat{\boldsymbol{\sigma}}\right)
$$

It is straightforward to show that $\hat{U}$ is periodic in $\phi$ with the period $2 \pi[13,[28]$. Accordingly we identify the adiabatic parameter space of the model with a two-dimensional plane $\left(B_{x}, B_{y}\right) \equiv(B \cos \phi, B \sin \phi)$.

We now diagonalize $\hat{U}$. First, $\hat{U}$ is expanded as $\hat{U}=$ $e^{-i \phi / 2}[\cos (\Delta / 2)-i \hat{\boldsymbol{\sigma}} \cdot \tilde{\boldsymbol{a}}]$, where

$$
\begin{gathered}
\Delta \equiv 2 \arccos \left(\cos \frac{\phi}{2} \cos \frac{B}{2}\right), \\
\tilde{\boldsymbol{a}} \equiv\left(\boldsymbol{e}_{\rho} \cos \frac{\phi}{2}-\boldsymbol{e}_{\phi} \sin \frac{\phi}{2}\right) \sin \frac{B}{2} \\
-\boldsymbol{e}_{z} \sin \frac{\phi}{2} \cos \frac{B}{2} .
\end{gathered}
$$

Because $\tilde{\boldsymbol{a}} \cdot \tilde{\boldsymbol{a}}=\sin ^{2}(\Delta / 2)$ holds, the eigenvalues of $\hat{U}$ become degenerate when $\sin (\Delta / 2)=0$ holds. Excluding the degeneracy points $\boldsymbol{B}=\mathbf{0}, 2 \pi \boldsymbol{e}_{x}, 4 \pi \boldsymbol{e}_{x}, \ldots$, we can normalize $\tilde{\boldsymbol{a}}$ :

$$
\boldsymbol{a} \equiv \tilde{\boldsymbol{a}} / \sin (\Delta / 2)
$$

We obtain the spectral decomposition of $\hat{U}$ in the form

$$
\hat{U}=z_{+} \hat{P}(\boldsymbol{a})+z_{-} \hat{P}(-\boldsymbol{a}),
$$

where $z_{ \pm} \equiv e^{-i\left(\phi \pm \frac{\Delta}{2}\right)}$ are eigenvalues, and

$$
\hat{P}(a) \equiv \frac{1+\boldsymbol{a} \cdot \hat{\boldsymbol{\sigma}}}{2},
$$

is a projection operator parameterized by a unit vector $\boldsymbol{a}$, which is called a (normalized) Bloch vector. Eq. (5) implies that $\hat{P}( \pm \boldsymbol{a})$ are the eigenprojectors of $\hat{U}$. In other words, for a given pair of $B_{x}$ and $B_{y}$, except at the degeneracy points, there are two normalized Bloch vectors $\pm \boldsymbol{a}$, which correspond to two eigenprojectors. We note that the spectral decomposition (Eq. (5)) is applicable to an arbitrary unitary Floquet operator or Hermitian Hamiltonian as long as the corresponding two level system has no spectral degeneracy. Hence the following argument is applicable to two-level systems in general.

Prior to the consideration of the exotic quantum holonomy in the kicked spin (1), a remark is due to the adiabatic time

quires either a level crossing or the divergence of eigenenergy [34]. To avoid complications from such singularities, we here examine periodically driven systems. 
evolution in periodically driven systems, for which parametric evolution of an eigenvector of the Floquet operator $\hat{U}$ describes the adiabatic time evolution, in place of a Hamiltonian, up to a phase factor. The dynamical phase is determined by a quasienergy [39] in place of eigenenergy. Proofs of the adiabatic theorem for Floquet systems are found in Refs. [40-42]. The corresponding adiabatic condition is governed by the gaps of quasienergies in place of eigenenergies [43].

We examine the adiabatic time evolution of the eigenprojector $\hat{P}(\boldsymbol{a})$ along a cycle $C$ in the $\left(B_{x}, B_{y}\right)$-plane. It is sufficient to examine the evolution of the normalized Bloch vector $\boldsymbol{a}$ instead of $\hat{P}(\boldsymbol{a})$ due to their equivalence. We depict the parametric evolution of $\boldsymbol{a}$ in Fig. 1 (a). Let $\boldsymbol{a}=\boldsymbol{a}_{0}$ at the initial point $\boldsymbol{B}_{0}$ on $C$. After a completion of the counterclockwise adiabatic rotation of $\boldsymbol{B}$ along $C, \boldsymbol{a}$ arrives at $-\boldsymbol{a}_{0}$ (see, Fig. 11a)), which implies that the final eigenprojector $\hat{P}\left(-a_{0}\right)$ is orthogonal to the initial one $\hat{P}\left(a_{0}\right)$. Hence $C$ induces the interchange of eigenprojectors $\hat{P}\left( \pm \boldsymbol{a}_{0}\right)$ resulting in the realization the eigenspace anholonomy. This fact is stable against the deformation of the adiabatic cycle, as long as $C$ encloses the origin only once. Let us next examine the case that $C$ does not enclose the origin. The simplest case is the one where $C$ start from $\boldsymbol{B}_{0}$ and keeps to stay $\boldsymbol{B}_{0}$, i.e., $C$ is a trivial cycle. The direction of the Bloch vector at the final point of the cycle agrees with the one at the initial point. Namely, the eigenprojector returns to the original one after the completion of the adiabatic cycle. This remains correct as long as $C$ does not enclose the origin $O$ in the $\left(B_{x}, B_{y}\right)$-plane. Also, the initial and final Bloch vectors are the same when $C$ encloses the origin even times.

\section{Eigenspace anholonomy as an anholonomy of an ordered set of mutually orthogonal projection operators}

Here, we propose a novel interpretation of the normalized Bloch vector $\boldsymbol{a}$ that allows the extension of our analysis to systems with an arbitrary number of levels. The central object is an ordered set of mutually orthogonal projection operators

$$
p \equiv\left(\left|\psi_{0}\right\rangle\left\langle\psi_{0}|,| \psi_{1}\right\rangle\left\langle\psi_{1}\right|\right)
$$

which can be specified by a normalized Bloch vector

$$
p(\boldsymbol{a})=(\hat{P}(\boldsymbol{a}), \hat{P}(-\boldsymbol{a}))
$$

A given pair of $B_{x}$ and $B_{y}$, except at the degeneracy points, specifies two normalized Bloch vectors $\pm \boldsymbol{a}$. One of them, say $\boldsymbol{a}$, precisely determines $p$. Another normalized Bloch vector $-\boldsymbol{a}$ correspond to another ordered set of projection operators $p(-\boldsymbol{a})=(\hat{P}(-\boldsymbol{a}), \hat{P}(\boldsymbol{a}))$, which is obtained by a permutation of the elements of $p(\boldsymbol{a})$. As for two-level systems, we can identify $p$ with a normalized Bloch vector $\boldsymbol{a}$, which helps our geometric intuition. The $p$-space of two-level systems is equivalent to the sphere $S^{2}$.

In terms of the ordered set of projectors $p$, the eigenspace anholonomy is the permutation of the elements of $p$ induced by an adiabatic cycle. For example, let us start an adiabatic cycle $C$ that enclose the origin of $\left(B_{x}, B_{y}\right)$-plane in Fig.11a). After the completion of the cycle $C$, the elements of $p$ are interchanged. In other words, $C$ corresponds to a permutation of the elements of $p$.

In this study, we make use of topological concepts of covering maps and homotopy groups [44, 45]. These concepts have been utilized, for example, in the studies of topological defects [35] and exceptional points [32]. A concise summary of the covering map is available in Ref. [32]. It will be shown that the resultant interpretation of the eigenspace anholonomy resembles Simon's interpretation of the phase holonomy in terms of vector bundles [2]. Although the following description is for the two-level kicked spin, the generalization to systems with an arbitrary number of levels is straightforward, as to be explained later.

Let us be more precise on the relationship between the adiabatic parameter space and $p$-space. Let $\mathcal{M}$ denote the adiabatic parameter space where the spectral degeneracy points are excluded. For a given point $x$ in $\mathcal{M}$, let $\mathcal{P}_{x}$ denote the set of two possible values of $p$. Since $\mathcal{P}_{x}$ may be regarded as a fiber, we denote the corresponding fiber bundle as $\mathcal{P}$. The projection from $\mathcal{P}$ to $\mathcal{M}$ is known as a covering map in topological analysis of manifolds [32, 44].

Despite the apparent similarity, there are several crucial differences between the fiber bundle appearing here and the fiber bundle interpretation of the phase holonomy. Among them, we point out the discreteness of the structure group of the fibers for the eigenspace anholonomy. This comes from the fact that the fiber bundle originates from the covering map [44], so that $p$ is "quantized" at a given point in $\mathcal{M}$. Contrarily, the structure group for the phase holonomy is mostly continuous [46].

For the kicked spin (1), $\mathcal{M}$ is $\left(B_{x}, B_{y}\right)$-plane excluding the degeneracy points. For a given point, say $\boldsymbol{B}$, in $\mathcal{M}$, there are two Bloch vectors, say $\pm \boldsymbol{a}$, so that a fiber $\mathcal{P}_{\boldsymbol{B}}$ consists of the two points $(\boldsymbol{B}, p( \pm \boldsymbol{a}))$. See, figure 2 (a). The corresponding structure group is the symmetric group of two elements (i.e., the group that contains all permutations for two items), and coincides with $\mathbb{Z}_{2}$. In order to simplify the following argument, we restrict $\mathcal{M}$ to be an annulus whose center is the origin in $\left(B_{x}, B_{y}\right)$-plane (e.g., $\frac{\pi}{2}<B<\frac{3 \pi}{2}$ ). (See, figures 1 a) and 2 (a)).

We now associate the time evolution of $p$ along an adiabatic cycle $C$ to the concept of lifting. For a given initial condition of $p$, the trajectory of $p$ induced by $C$ essentially determines the lifting $\tilde{C}$ of $C$ to $\mathcal{P}$ [46]. The situation is visualised in Fig. 2 (a). As for the kicked spin, for a given initial point of $C$, say, $\boldsymbol{B}_{0}$, there are two possible normalized Bloch vectors $\pm \boldsymbol{a}_{0}$, which provides two different lifts of $C$. Suppose $\boldsymbol{B}_{0}$ is slightly varied. Correspondingly, the Bloch vectors $\pm \boldsymbol{a}_{0}$ are smoothly and slightly deformed. The repetition of this procedure determines the two lifts $\tilde{C}_{ \pm}$of $C$.

The lift $\tilde{C}$ tells us whether the eigenprojectors in $p$ are interchanged by the adiabatic cycle $C$. When $\tilde{C}$ is a closed path, each eigenprojector in $p$ also draws a closed path. This implies the absence of the eigenspace anholonomy. On the other hand, when $\tilde{C}$ is open, the initial and the final points of an eigenprojector are different, which is the case in which the eigenspace anholonomy occurs. Furthermore, the destination of $\tilde{C}$ precisely describes the permutation among the eigenprojectors. 
Let $\phi_{C}(p)$ denote the final point of the lifted path $\tilde{C}$ of $C$, where $p$ is the initial point of $\tilde{C}$ path. Our question of the eigenspace anholonomy is now casted into the problem of determining $\phi_{C}$. The mapping $\phi_{C}$ is called a monodromy action in the analysis of covering map [44]. For a given initial point in $\mathcal{P}$, let $\Phi$ denote the set of $\phi_{C}$ generated by all possible adiabatic cycles. $\Phi$ is called the automorphism group of the covering [44], and is the counterpart of the holonomy group for the phase holonomy.

Not every detail of $C$ is required to determine $\phi_{C}$. This is to be expected from the fact that $\phi_{C}$ corresponds to a permutation, and thus is "quantized". In the analysis of covering map, the homotopy classification of $C$ plays the central role [32, 44]. We say that a path $C$ is homotopic to another path $C^{\prime}$, when $C$ can be smoothly deformed to $C^{\prime}$ with the initial and final points kept unchanged. Let $[C]$ denote the class of paths that are homotopic to $C$ [44]. Since $\phi_{C}$ and $\phi_{C^{\prime}}$ are the same as long as $C$ is homotopic to $C^{\prime}$ because of the homotopy lifting property, the quantity $\phi_{C}$ may be denoted as $\phi_{[C]}[44]$. Hence, it suffices to examine $\phi_{[C]}$ with $[C]$ belonging to the first fundamental group of $\mathcal{M}$, i.e., $[C] \in \pi_{1}(\mathcal{M})$.

It is important, at the same time, to observe that $\phi_{[C]}$ describes the identical permutation if and only if the lifted cycle $\tilde{C}$ of $C$ is homotopic to a closed path in $\mathcal{P}$, i.e., $[\tilde{C}] \in \pi_{1}(\mathcal{P})$.

In order to express this condition in terms of $\mathcal{M}$, we consider the projection of all the elements $\pi_{1}(\mathcal{P})$ to $\mathcal{M}$. We denote the resultant set as $H$, which is a subset of $\pi_{1}(\mathcal{P})$ and is called an isotropy group. The quotient space $\pi_{1}(\mathcal{M}) / H$ precisely classifies $\phi_{[C]}$, i.e., $\Phi \simeq \pi_{1}(\mathcal{M}) / H$, according to the covering automorphism group structure theorem (Ref [44], Theorem 12.7).

For example, the kicked spin (1) has $\pi_{1}(\mathcal{M})=$ $\left\{[e],[\alpha],\left[\alpha^{2}\right], \ldots\right\}$, where $e$ is homotopic to a point and $\alpha$ encloses the origin $O$ once in $\mathcal{M}$ (see, figure 2). On the other hand, $\pi_{1}(\mathcal{P})=\left\{\left[e^{\prime}\right],[\beta],\left[\beta^{2}\right], \ldots\right\}$ where $e^{\prime}$ is homotopic to a point, and $\beta$ encloses the "hole" in $\mathcal{P}$. Because the projection of $\beta$ to $\mathcal{M}$ is homotopic to $\alpha^{2}$, we find $H=\left\{[e],\left[\alpha^{2}\right],\left[\alpha^{4}\right], \ldots\right\}$. Hence, $\Phi=\pi_{1}(M) / H$ consists of two classes. One corresponds to the cycles that encloses enclose $O$ even times, and makes $\phi_{[C]}$ the identity. The other is composed by the cycles that enclose $O$ odd times to make $\phi_{[C]}$ the cyclic permutation of the two items in $p$. Hence we conclude that $\Phi$, the automorphism group of the covering, is equivalent with $\mathbb{Z}_{2}$, the cyclic group whose order is 2 .

\section{Definition of cycles by quantum dynamical variables in- stead of c-number parameters}

So far, cycles are parameterized by the adiabatic parameters $\left(B_{x}, B_{y}\right)$. This has been a common definition in the previous studies of the exotic quantum holonomy [3, 27]. Instead, we propose a way to define the cycles only in terms of quantum dynamical variables. The aim here is twofold. One is to complete a geometrical view of the eigenspace anholonomy. Another is to extend the exotic quantum holonomy into nonadiabatic cycles, which will be examined in the next section. To achieve this, we introduce a set of mutually orthogonal eigenprojectors

$$
b \equiv\left\{\left|\psi_{0}\right\rangle\left\langle\psi_{0}|,| \psi_{1}\right\rangle\left\langle\psi_{1}\right|\right\},
$$

where the order of the projector are disregarded.

As for the two level systems, we obtain a geometric interpretation of $b$ with the help of a normalized Bloch vector $\boldsymbol{a}$

$$
b(\boldsymbol{a})=\{\hat{P}(\boldsymbol{a}), \hat{P}(-\boldsymbol{a})\},
$$

which agrees with $b(-\boldsymbol{a})$, since the order of the elements in $b$ is ignored. In other words, we identify $\boldsymbol{a}$ and $-\boldsymbol{a}$ in the specification of $b$. In geometry, the identification of antipodal points on the sphere $S^{2}$ leads to the real projective plane $\mathbb{R} P^{2}$ [35, 45]. Hence we identify $b$ with a point, which we denote as $\boldsymbol{n}$, in the projective plane. In Fig. 1 (b), $\boldsymbol{n}$ is depicted in the $\left(B_{x}, B_{y}\right)$ plane. We note that $\boldsymbol{n}$ is single-valued here. Still, we have a singularity at the origin $O$, where the value of $\boldsymbol{n}$ cannot be determined. This resembles a disclination of nematic liquid crystals [36]. In the studies of nematic liquid crystals, $\boldsymbol{n}$ is called as a director, or a headless vector [36]. The disclination in nematics is a discontinuity of $\boldsymbol{n}$-field in three-dimensional space and the discontinuity may extends along a line [35, 45]. In this sense, the disclination is distinct from the effective monopole induced by the phase holonomy [1]. We use $\boldsymbol{n}$, or equivalently $b$, to define an adiabatic cycle $C$. For the quantum kicked spin (Eq. (1)), we regard that the path $C$ resides in the $\boldsymbol{n}$-space rather than in the $\left(B_{x}, B_{y}\right)$-plane,

It is possible to examine all two-level systems that have no spectral degeneracy with $\mathbb{R} P^{2}$. This is because the director space $\mathbb{R} P^{2}$ can parameterize an arbitrary adiabatic cycle of two level systems including the kicked spin- $\frac{1}{2}$, as long as the adiabatic cycle do not encounter any spectral degeneracy. We have a complete classification of the adiabatic cycles of nondegenerate two-level systems in following manner.

Let $\mathcal{B}$ denote the adiabatic parameter space $\mathbb{R} P^{2}$. Now $\mathcal{P}$ is equivalent to the whole sphere $S^{2}$. There is a covering map from $\mathcal{P}$ to $\mathcal{B}$, in the sense that the inverse of the covering map of $\boldsymbol{n}$ is the set of two Bloch vectors $\pm \boldsymbol{n}$. The fundamental group of $\mathcal{P}$ is $\pi_{1}(\mathcal{P})=\left\{\left[e^{\prime}\right]\right\}$, where $e^{\prime}$ is a cycle homotopic to a point in $\mathcal{P}$. The simplicity of $\pi_{1}(\mathcal{P})$ compared to the previous case is the reflection of the fact that $\mathcal{P}$ is "larger" than the previous one, as we have "extended" the base space from $\mathcal{M}$, an annulus, to $\mathbb{R} P^{2}$. On the other hand, the fundamental group of the adiabatic parameter space is $\pi_{1}\left(\mathbb{R} P^{2}\right)=\{[e],[\gamma]\}$, where $e$ is homotopic to a point in $\mathbb{R} P^{2}$ and $\gamma$ a quantity not homotopic to $e$. We note that $\gamma^{2}$, i.e., the repetition of $\gamma$ twice is homotopic to $e$ (see, Fig. 2(b)). Because $\mathcal{P}$ is a universal cover of $\mathcal{B}$, there are only two classes of monodromy actions $\phi_{[C]}$, which is either $\phi_{[e]}$ or $\phi_{[\gamma]}$. The former and latter cases correspond to the absence and the presence of the eigenspace anholonomy respectively (see Fig. 22(b) again). We conclude that $\Phi=\pi_{1}\left(\mathbb{R} P^{2}\right)$ is equivalent with $\mathbb{Z}_{2}$.

\section{Nonadiabatic extension}

In our geometrical treatment of the eigenspace anholonomy so far, there has been no mention on the scale of temporal variations for the director $\boldsymbol{n}$, nor for the set of projectors $b$. In the 


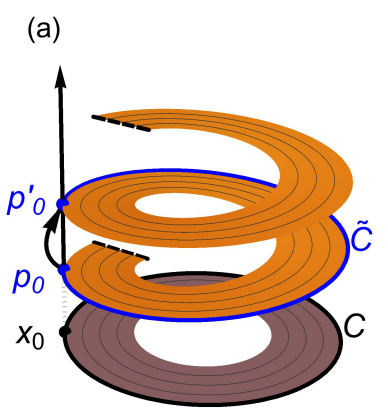

(b)

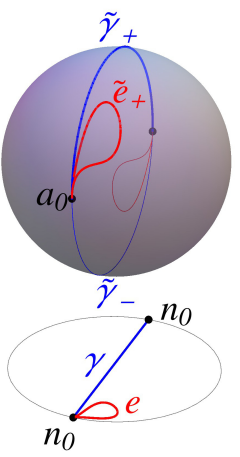

Figure 2: The covering map structures of two-level systems (schematic). (a) The adiabatic space $\mathcal{M}$ (bottom annulus) of the kicked spin- $\frac{1}{2}(1)$ is chosen so as to avoid degeneracy points (e.g., $\frac{\pi}{2}<B<\frac{3 \pi}{2}$ in Figure 1 ). Since two edges depicted by dashed lines of $\mathcal{P}$ (the winding strip in the above) should be identified, $\mathcal{P}$ is a "double-winding" strip above $\mathcal{M}$. The projection from $\mathcal{P}$ to $\mathcal{M}$ is a covering map. The adiabatic cycle $C$ starts from $x_{0}$ and winds $\mathcal{M}$ in the counterclockwise direction. The corresponding lifting $\tilde{C}$ delivers $p_{0}$ to $p_{0}^{\prime}$. Accordingly the monodromy action $\phi_{C}$ describe the motion in the fiber direction. (b) Adiabatic cycles of the two-level systems without spectral degeneracies can be parameterized by the projective plane $\mathcal{M}$, which is equivalent with $\mathbb{R} P^{2}$, shown in the bottom part. Two cycles (closed paths) $e$ and $\gamma$ are shown there. Two filled circles corresponding to $\boldsymbol{n}_{0}$, which is the initial point of these cycles, are identical in $\mathbb{R} P^{2}$. The cycle $e$ is homotopic to a zero-length cycle, and is not homotopic to $\gamma$. In the top, the lifts of $e$ and $\gamma$ to the $p$-space, which is equivalent to $S^{2}$, are shown. Because $S^{2}$ doubly covers $\mathbb{R} P^{2}$, there are two normalized Bloch vectors $\pm \boldsymbol{a}_{0}$ for a given director $\boldsymbol{n}_{0}$. Also, each cycle has two lifts (thick and dashed curves). The lifts $\tilde{e}_{ \pm}$of $e$ are closed, signifying the absence of eigenspace anholonomy. On the other hand, the lifts $\tilde{\gamma}_{ \pm}$are open. Along the adiabatic cycle $\gamma$, the initial point $\boldsymbol{a}_{0}$ of $\tilde{\gamma}_{+}$is transposed to $-\boldsymbol{a}_{0}$, which is the initial point of $\tilde{\gamma}_{-}$, and vice versa.

adiabatic regime, we regard $\boldsymbol{n}$ and $b$ as adiabatic parameters. We can also consider the case in which $\boldsymbol{n}$ and $b$ are quantum dynamical variables. In this case, the time evolution defined by quantum theory naturally induces the time evolution of $\boldsymbol{n}$ and $b$ which may or may not be adiabatic. We can then define the cycles made out of nonadiabatic time evolution of $\boldsymbol{n}$ and $b$.

We give an example of a two level system described by a time-periodic Hamiltonian, whose time dependence may or may not be adiabatic. Let $\hat{F}$ denote a Floquet operator, which describes the time evolution during its period. We suppose that $\hat{F}$ has two eigenvectors $|0\rangle$ and $|1\rangle$. The trajectory of the Bloch vector of $(|0\rangle\langle 0|| 1\rangle,\langle 1|)$ forms a closed curve, and the trajectory of director of $\{|0\rangle\langle 0|| 1\rangle,\langle 1|\}$ is homotopic to a point in $\mathbb{R} P^{2}$, implying the absence of anholonomy.

We show that the nonadiabatic anholonomy occurs if $\hat{F}$ has two eigenvalues $\exp \{-i(\epsilon \pm \pi / 2)\}$, where $\epsilon$ is an arbitrary real number. Let us write two eigenstates of $\hat{F}$ as $|0\rangle$ and $|1\rangle$, namely

$$
\hat{F}|0\rangle=e^{-i(\epsilon+\pi / 2)}|0\rangle, \quad \hat{F}|1\rangle=e^{-i(\epsilon-\pi / 2)}|1\rangle .
$$

A pair of normalized vectors

$$
| \pm\rangle \equiv \frac{1}{\sqrt{2}}(|0\rangle \pm|1\rangle)
$$

exhibit nonadiabatic anholonomy. This is because, from Eq. (11), $\hat{F}|+\rangle=e^{-i(\epsilon+\pi / 2)}|-\rangle$ and $\hat{F}|-\rangle=e^{-i(\epsilon+\pi / 2)}|+\rangle$ hold. In other words, the trajectory of the Bloch vectors of

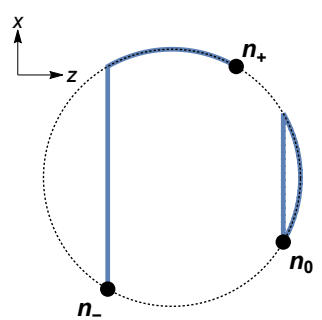

Figure 3: Nonadiabatic cycles in the director space $\mathbb{R} P^{2}$ for the kicked spin (Eq. (13) with $B=\pi / 3$. Trajectories of director is depicted in $\left(n_{z}, n_{x}\right)$-plane. The unit circle in $\left(n_{z}, n_{x}\right)$-plane is also shown (dotted). $\boldsymbol{n}_{0}$ correspond to the set of eigenprojectors $\{|0\rangle\langle 0|| 1\rangle,\langle 1|\}$ of $\hat{F}$. The trajectory $C_{0}$ that starts from $\boldsymbol{n}_{0}$ is homotopic to a contractable loop $e$ (see, Fig. 2 b)). On the other hand, let $C_{+}$be the cycle whose initial point $\boldsymbol{n}_{+}$correspond to the set of projectors $\{|+\rangle\langle+||-\rangle,\langle-|\}$ (see, Eq. (12)). The end point of $C_{+}$is $\boldsymbol{n}_{-}$, which is equivalent with $\boldsymbol{n}_{+}$. Note that $C_{+}$is not homotopic to $C_{0}$. Suppose the system is initially at $|+\rangle$. Due to the time evolution along $C_{+}$, the system arrives at $|-\rangle$ to exhibit the nonadiabatic anholonomy.

$(|+\rangle\langle+||-\rangle-,\mid)$ draws an open curve during the period of the Hamiltonian. The corresponding trajectory of the director must be closed and homotopic to $\gamma$ in $\mathbb{R} P^{2}$.

An example of $\hat{F}$ is provided by the kicked spin- $\frac{1}{2}$, which is described by the following Hamiltonian

$$
\hat{H}(t) \equiv \frac{\pi}{2} \hat{\sigma}_{z}+\frac{B}{2} \hat{\sigma}_{y} \sum_{n=-\infty}^{\infty} \delta(t-n),
$$

where we assume $0<B<\pi$. The period of $H(t)$ is unity. The corresponding Floquet operator is $\hat{F}=$ $\exp \left(-i \pi \hat{\sigma}_{z} / 2\right) \exp \left(-i B \hat{\sigma}_{y} / 2\right)$, whose normalized eigenvectors are

$$
|0\rangle \equiv\left(\begin{array}{c}
\cos \frac{B}{4} \\
-\sin \frac{B}{4}
\end{array}\right), \quad|1\rangle \equiv\left(\begin{array}{l}
\sin \frac{B}{4} \\
\cos \frac{B}{4}
\end{array}\right) .
$$

Indeed, they satisfy Eq. (11) with $\epsilon=0$. We depict the trajectories of the directors of two sets of projectors $\{|0\rangle\langle 0|| 1\rangle,\langle 1|\}$ and $(|+\rangle\langle+||-\rangle,\langle-|)$ during the unit time interval of the Hamiltonian (13) in $\mathbb{R} P^{2}$ in Figure 3

\section{6. $N$-dimensional extension}

We close our argument by outlining the extension to cases that involves an arbitrary number, say $N$, of levels.

First, let us note that the definitions of $p$ and $b$ (Eqs. (7) and (9)), which consist of mutually orthogonal projection operators, and also the definitions of $\mathcal{P}$ and $\mathcal{B}$ are applicable to an arbitrary $N$. We do not attempt, however, to extend the concepts of Bloch vectors and directors for now. We emphasize that $\mathcal{P}$ is a covering space of $\mathcal{B}$ [32, 44], irrespective of the value of $N$. The quantity $\mathcal{P}$ is referred to as a flag manifold [47, 48].

It is still an open problem to provide a complete classification of the cycles for an arbitrary $N$-level system. This is due to the difficulty in identifying the fundamental groups of $\mathcal{P}$ and $\mathcal{M}$ for the $N$-level case.

Non the less, it is possible to provide a working example of the present formulation with an arbitrary $N$ if we choose appropriate sub-family of the general $N$-level systems. Let us 
examine a family of quantum maps whose unit time evolution operator is given by $\hat{U}(\lambda) \equiv \hat{U}_{0} \exp (-i \lambda|v\rangle\langle v|)$, in which $\lambda$ is an adiabatic parameter, $\hat{U}_{0}$ a nondegenerate unitary operator, and $|v\rangle$ a normalized vector. We also assume that $|v\rangle$ is not an eigenvector of $\hat{U}_{0}$. The following is a summary of the result of Ref. [14] on the exotic quantum holonomy in this model. Let $|n\rangle$ denote the $n$-th eigenstate of $\hat{U}_{0}$, where the quantum number $n$ is assigned in the increasing order of quasienergy. Since $\hat{U}(\lambda)$ is $2 \pi$-periodic in $\lambda$, the adiabatic parameter space $\mathcal{M}$ is identified with a circle $S^{1}$. Let $C$ denote an adiabatic cycle where $\lambda$ is increased from 0 to $2 \pi$. It is shown in Ref. [14] that the eigenspace anholonomy is induced by $C$ because $C$ delivers $|n\rangle$ to $|n+1\rangle$.

Applying the present formulation to this model, we first note that we have $\pi_{1}(\mathcal{M})=\left\{[e],[\alpha],\left[\alpha^{2}\right], \ldots\right\}$, where $e$ and $\alpha$ are a point and a cycle in $\mathcal{M}$, as $\mathcal{M}$ is regarded as $S^{1}$ [45]. Also we find $\pi_{1}(\mathcal{P})=\left\{\left[e^{\prime}\right],[\beta],\left[\beta^{2}\right], \ldots\right\}$, where $e^{\prime}$ is homotopic to a point, and $\beta$ encloses the "hole" in $\mathcal{P}$ (cf. Figure 2(a)). The $N$ dependence appears in the fact that the projection of $\beta$ is homotopic to $\alpha^{N}$. Hence the isotropy group is $H=\left\{[e],\left[\alpha^{N}\right],\left[\alpha^{2 N}\right], \ldots\right\}$. We conclude $\Phi\left(=\pi_{1}(M) / H\right)$ is equivalent with $\mathbb{Z}_{N}, N$-th cyclic group.

\section{Discussion}

We discuss the relationship of this work to the previous works on the eigenspace anholonomy. In the present formulation, we have identified the layers in the hierarchy of the quantum holonomy, which consists of the $b$-space, $p$-space and the space consists of frames $\left(\left|\psi_{0}\right\rangle,\left|\psi_{1}\right\rangle, \ldots\right)$. A lift of a closed cycle of $b$ to the $p$-space involves the anholonomy in $p$. In contrast, a lift of a path in $p$-space to the frame space involves the phase holonomy. In particular, Aharonov-Anandan phase is induced by the cycle in $p$-space. On the other hand, the phase holonomy associated with a open path in $p$-space correspond to the off-diagonal geometric phase [49, 50]. In a gauge theoretical approach introduced in Ref. [27], the eigenspace anholonomy and the off-diagonal geometric phase are treated together. These two concepts are disentangled and assigned to the different layers through the present formulation.

A remark is due to the relationship between the exotic quantum holonomy and Kato's exceptional point (EP), which is a branch point of the Riemann surface of eigenenergies, in nonHermitian quantum theory [6, 7]. The adiabatic time evolution under the presence of the eigenspace anholonomy resembles a parametric evolution that encloses an EP, in the sense that these evolutions permutate eigenspaces. An analytic continuation of adiabatic cycle in Hermitian Hamiltonian and unitary Floquet systems has enabled to interpret the exotic quantum holonomy as the result of parametric encirclement of EP in the complex plane [30, 31]. Although such a correspondence is valid only when an analytic continuation of the adiabatic cycle is available, the topological formulation is applicable regardless of the analytic continuation. Also, we do not know how the non-adiabatic extension of exotic quantum holonomy can be associated with EPs. On the other hand, we remind the readers that the relationship between the phase holonomy and EPs is established through the analysis of the Riemann surface of (quasi-)eigenenergy [32]. Because the covering space structure naturally resides in the Riemann surfaces, an extension of the present approach to non-Hermitian systems should be of interest.

\section{Acknowledgments}

AT wish to thank Professor Ali Mostafazadeh for a useful conversation and Professor Akira Shudo for comments. This research was supported by the Japan Ministry of Education, Culture, Sports, Science and Technology under the Grant number 24540412.

\section{References}

[1] M. V. Berry, Quantal phase factors accompanying adiabatic changes, Proc. R. Soc. London A 392 (1984) 45-57.

[2] B. Simon, Holonomy, the Quantum Adiabatic Theorem, and Berry's Phase, Phys. Rev. Lett. 51 (24) (1983) 2167-2170.

[3] T. Cheon, Double spiral energy surface in one-dimensional quantum mechanics of generalized pointlike potentials, Phys. Lett. A 248 (1998) 285289.

[4] F. Wilczek, A. Zee, Appearance of gauge structure in simple dynamical systems, Phys. Rev. Lett. 52 (1984) 2111-2114.

[5] R. Uzdin, A. Mailybaev, N. Moiseyev, On the observability and asymmetry of adiabatic state flips generated by exceptional points, Journal of Physics A: Mathematical and Theoretical 44 (43) (2011) 435302.

[6] T. Kato, Perturbation Theory for Linear Operators, corrected printing of the second Edition, Springer-Verlag, Berlin, 1980, Ch. II.

[7] See, e.g., N. Moiseyev, Non-Hermitian Quantum Mechanics (Cambridge Univ. Press, New York, 2011).

[8] M. V. Berry, R. Uzdin, Slow non-Hermitian cycling: exact solutions and the Stokes phenomenon, Journal of Physics A: Mathematical and Theoretical 44 (43) (2011) 435303.

[9] C. Dembowski, H.-D. Gräf, H. Harney, A. Heine, W. Heiss, H. Rehfeld, A. Richter, Experimental observation of the topological structure of exceptional points, Phys. Rev. Lett. 86 (2001) 787-790.

[10] S.-B. Lee, J. Yang, S. Moon, S.-Y. Lee, J.-B. Shim, S. Kim, J.-H. Lee, K. An, Observation of an exceptional point in a chaotic optical microcavity, Phys. Rev. Lett. 103 (2009) 134101.

[11] T. Cheon, T. Fülöp, I. Tsutsui, Symmetry, duality and anholonomy of point interaction in one dimension, Ann. Phys. (N.Y.) 294 (2001) 1-23.

[12] I. Tsutsui, T. Fülöp, T. Cheon, Moebius structure of the spectral space of schroedinger operators with point interaction, J. Math. Phys. 42 (2001) 5687-5697.

[13] A. Tanaka, M. Miyamoto, Quasienergy anholonomy and its application to adiabatic quantum state manipulation, Phys. Rev. Lett. 98 (2007) 1604071-160407-4.

[14] M. Miyamoto, A. Tanaka, Cheon's anholonomies in Floquet operators, Phys. Rev. A 76 (2007) 042115-1-042115-9.

[15] A. Tanaka, S. W. Kim, T. Cheon, Eigenvalue and eigenspace anholonomies in hierarchical systems, Europhys. Lett. 96 (2011) 10005p1-10005-p6.

[16] S. Ohya, Parasupersymmetry in quantum graphs, Ann. Phys. (NY) 331 (2013) 299.

[17] S. Ohya, Non-abelian monopole in the parameter space of point-like interactions, Ann. Phys. (NY) 351 (2014) 900.

[18] T. Cheon, A. Tanaka, O. Turek, Examples of quantum holonomy with topology changes, Acta Polytechnica 53 (2013) 410.

[19] E. H. Lieb, W. Liniger, Exact Analysis of an Interacting Bose Gas. I. The General Solution and the Ground State, Phys. Rev. 130 (4) (1963) 16051616.

[20] T. Ichikawa, I. Tsutsui, N. Yonezawa, Equation of state for the onedimensional attractive $\delta$-potential Bose gas in the weak-coupling regime, Phys. Rev. A 86 (2012) 015602. 
[21] M. Olshanii, Atomic Scattering in the Presence of an External Confinement and a Gas of Impenetrable Bosons, Phys. Rev. Lett. 81 (1998) 938.

[22] E. Haller, M. Gustavsson, M. J. Mark, J. G. Danzl, , R. Hart, G. Pupillo, H.-C. Nägerl, Realization of an Excited, Strongly Correlated Quantum Gas Phase, Science 325 (2009) 1224.

[23] N. Yonezawa, A. Tanaka, T. Cheon, Quantum holonomy in the LiebLiniger model, Phys. Rev. A 87 (2013) 062113.

[24] A. Tanaka, K. Nemoto, Adiabatic quantum computation along quasienergies, Phys. Rev. A 81 (2010) 022320-1-022320-8.

[25] K. Fujikawa, Geometric phases and hidden local gauge symmetry, Phys. Rev. D 72 (2005) 025009.

[26] K. Fujikawa, Geometric phases for mixed states and decoherence, Ann. Phys. (N.Y.) 322 (2007) 1500-1517.

[27] T. Cheon, A. Tanaka, New anatomy of quantum holonomy, Europhys. Lett. 85 (2009) 20001-p1-20001-p5.

[28] A. Tanaka, T. Cheon, A unified theory of quantum holonomies, Ann. Phys. (N.Y.) 324 (2009) 1340-1359.

[29] D. Viennot, Geometric phases in adiabatic Floquet theory, Abelian gerbes and Cheon's anholonomy, J. Phys. A. 42 (2009) 395302, (22pp).

[30] S. W. Kim, T. Cheon, A. Tanaka, Exotic quantum holonomy induced by degeneracy hidden in complex parameter space, Phys. Lett. A 374 (2010) 1958-1961.

[31] A. Tanaka, N. Yonezawa, T. Cheon, Exotic quantum holonomy and nonHermitian degeneracies in the two-body Lieb-Liniger model, Journal of Physics A: Mathematical and Theoretical 46 (31) (2013) 315302.

[32] H. Mehri-Dehnavi, A. Mostafazadeh, Geometric phase for non-hermitian hamiltonians and its holonomy interpretation, J. Math. Phys. 49 (2008) 082105 .

[33] A. Tanaka, T. Cheon, S. W. Kim, Gauge invariants of eigenspace and eigenvalue anholonomies: Examples in hierarchical quantum circuits, Journal of Physics A: Mathematical and Theoretical 45 (33) (2012) 335305-1-335305-20.

[34] T. Cheon, A. Tanaka, S. W. Kim, Exotic quantum holonomy in Hamiltonian systems, Phys. Lett. A 374 (2009) 144-149.

[35] N. D. Mermin, The topological theory of defects in ordered media, Rev. Mod. Phys. 51 (1979) 591

[36] P. G. de Gennes, J. Prost, The Physics of Liquid Crystals, 2nd Edition, Oxford University Press, Oxford, 1993, Ch. 1.

[37] Y. Aharonov, J. Anandan, Phase change during a cyclic quantum evolution, Phys. Rev. Lett. 58 (1987) 1593-1596.

[38] M. Combescure, Spectral properties of a periodically kicked quantum hamiltonian, J. Stat. Phys. 59 (1990) 679-690.

[39] Y. B. Zel'dovich, The quasienergy of a quantum-mechanical system subjected to a periodic action, J. Exp. Theor. Phys. 24 (6) (1967) 1006-1008.

[40] R. H. Young, W. J. Deal Jr., Adiabatic Response to an Oscillatory Field, J. Math. Phys. 11 (11) (1970) 3298-3306.

[41] A. Dranov, J. Kellendonk, R. Seiler, Discrete time adiabatic theorems for quantum mechanical systems, Journal of Mathematical Physics 39 (3) (1998) 1340.

[42] A. Tanaka, Adiabatic theorem for discrete time evolution, Journal of the Physical Society of Japan 80 (2011) 125002-1-125002-2.

[43] H. Breuer, M. Holthaus, Adiabatic processes in the ionization of highly excited hydrogen atoms, Z. Phys. D 11 (1989) 1-14.

[44] J. M. Lee, Introduction to Topological Manifolds, 2nd Edition, Vol. 202 of Graduate Texts in Mathematics, Springer, New York, 2011.

[45] M. Nakahara, Geometry, Topology and Physics, IOP Publishing Ltd, Bristol, 1990

[46] A. Bohm, A. Mostafazadeh, H. Koizumi, Q. Niu, Z. Zwanziger, The Geometric Phase in Quantum Systems, Springer, Berlin, 2003.

[47] M. Adelman, J. V. Corbett, C. A. Hurst, The geometry of state space, Found. Phys. 23 (2) (1993) 211.

[48] I. Bengtsson, K. Życzkowski, Geometry of Quantum States, Cambridge University Press, Cambridge, 2006, Ch. 4.

[49] N. Manini, F. Pistolesi, Off-Diagonal Geometric Phases, Phys. Rev. Lett. 85 (2000) 3067-3071.

[50] N. Mukunda, Arvind, S. Chaturvedi, R. Simon, Bargmann invariants and off-diagonal geometric phases for multilevel quantum systems: A unitarygroup approach, Phys. Rev. A 65 (2001) 012102-1-012102-10. 